\title{
Finite group torsors for the qfh topology
}

\author{
J.F. Jardine
}

December 2, 2000

\section{Introduction}

Suppose that $S$ is a Noetherian scheme and $G$ is a group scheme which is finite and étale over $S$. The purpose of this note is to show that torsors in Voevodsky's qfh topology for such group schemes $G$ coincide up to isomorphism with torsors induced up from the étale topology, in the sense that the canonical function

$$
H_{e t}^{1}(S, G) \rightarrow H_{q f h}^{1}(S, G)
$$

between the associated non-abelian cohomology objects is a bijection. This is the main result of this paper and appears here as Theorem 12.

This theorem is the non-abelian analogue of a result of Voevodsky [8, 3.4.4], which asserts the existence of isomorphisms

$$
H_{e t}^{n}(S, A) \cong H_{q f h}^{n}(S, A), \quad n \geq 0,
$$

for abelian sheaves $A$ which are locally constant for the étale topology.

The proof of Voevodsky's result is a Leray spectral sequence argument which uses the site of finite surjective covers for strict Hensel local rings. The present proof also makes use of this site, but in the context of simplicial sheaf homotopy theory. In particular, the main result can be rephrased as the assertion that the canonical map of simplicial sheaves $B G \rightarrow B S t_{q f h} G$ taking values in the classifying object of the qfh stack completion of $G$ [3] is a local weak equivalence for the étale topology. The main technical devices include the assertion (Proposition 8) that degreewise finite constant simplicial sheaves satisfy a qfh descent condition on strict local Hensel rings, and also that inverse images of qfh sheaves over strict Hensel local rings can be computed via filtered colimits (Lemma 11). This last thing is a filtered colimit of finite sites argument which should be familiar from étale cohomology theory, but has been written here in some detail.

The range of applications here includes all finite Galois groups $G$ which occur in the context of the transfer construction in [4]. In that context the present result is saying that there are no exotic qfh torsors which arise from rational points of quotient stacks associated to finite normalizations. 


\section{Results}

Suppose that $S$ is a Noetherian scheme, and let $\left.S c h\right|_{S}$ denote the category of $S$-schemes of finite type. Recall [8] that the qfh topology for $S$ is generated by all finite families $p: U_{i} \rightarrow X$ of quasi-finite morphisms such that their sum $\sqcup_{i} U_{i} \rightarrow X$ is a universal topological epimorphism. The collection of qfh covering families includes all finite families of flat (and hence open, or étale) maps. Recall that a map of schemes is quasi-finite if it is of finite type and has discrete fibres.

To illustrate, suppose that $p_{i}: U_{i} \rightarrow X$ is a qfh covering family, and choose a finite affine open cover $S p\left(A_{j}\right)$ of the base scheme $X$. Then the collection of maps $S p\left(A_{j}\right) \times_{X} U_{i} \rightarrow S p\left(A_{j}\right)$ is a qfh covering family for $S p\left(A_{j}\right)$. Further, if $S p\left(B_{k, j}\right)$ is a finite affine open cover for $S p\left(A_{j} \times_{X} U_{i}\right)$, then the collection of maps $S p\left(B_{k, j}\right) \rightarrow S p\left(A_{j}\right)$ is a qfh covering family for $S p\left(A_{j}\right)$, and so the collection of all composites

$$
S p\left(B_{k, j}\right) \rightarrow S p\left(A_{j}\right) \hookrightarrow X
$$

is a qfh covering family for $X$. All such composites are separated maps, so we have shown that any qfh cover can be refined by a qfh cover consisting of separated morphisms. This is a rather primitive example of the refinements that can be found for qfh covers - many other examples are given in [8].

The notation $\left(\left.S c h\right|_{S}\right)_{q f h}$ will be reserved for the site of $X$-schemes of finite type, with the qfh topology. Similarly, $\left(\left.S c h\right|_{S}\right)_{e t}$ denotes the site of $S$-schemes of finite type, endowed with the étale topology. Following [8], LP denotes the associated sheaf of a presheaf $P$ for the qfh topology. Recall that representable presheaves are not necssarily sheaves for this topology; in particular [8, 3.2.11], if $Y$ is normal and connected and $X$ is any $S$-scheme of finite type, then the set of $Y$-sections $L(X)(Y)$ of the qfh sheaf associated to the presheaf represented by $X$ is a filtered colimit of sets $\operatorname{hom}_{X}\left(Y_{L}, X\right)$ of morphisms defined on normalizations $Y_{L}$ of $Y$, indexed by the category of purely inseparable field extensions $L / k(Y)$.

Lemma 1. Suppose that $k$ is a separably closed field, and suppose that $P$ is a presheaf on $\left(\left.S c h\right|_{k}\right)_{q f h}$ such that any finite purely inseparable extension $L / k$ induces an isomorphism $P(k) \cong P(L)$. Then the associated sheaf map $\eta: P \rightarrow$ $L P$ induces a bijection $P(k) \cong L P(k)$.

Proof. The associated sheaf map $\eta$ is both a local epi and a local monic. For any element $\alpha \in L P(k)$ there is a quasi-finite universal topological epimorphism $\phi: V \rightarrow S p(k)$ such that $\phi^{*}(\alpha)$ lifts to $P(V)$ The function field $k(x)$ of any point $x \in V$ is a finite algebraic extension of $k$, and hence a purely inseparable extension since $k$ is separably closed. It therefore follows from the commutative diagram

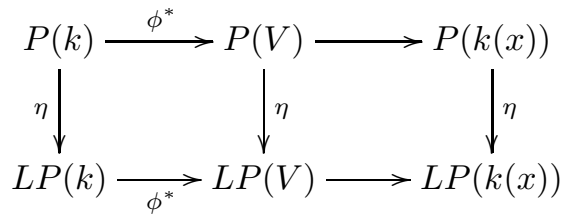


and the fact that both horizontal compositions are isomorphisms that $\alpha$ is in the image of $\eta: P(k) \rightarrow L P(k)$. Similarly, if $\eta(\alpha)=\eta(\beta)$ for $\alpha, \beta \in P(k)$, there there is a $\phi: V \rightarrow S p(k)$ as above such that $\phi^{*}(\alpha)=\phi^{*}(\beta)$ in $P(V)$. But then $\alpha$ and $\beta$ go to the same element in $P(k(x))$ so that $\alpha=\beta$.

Corollary 2. The functor $\operatorname{hom}(L S p(k)$,$) takes sheaf epis to surjective func-$ tions on the site $\left(\left.S c h\right|_{k}\right)_{q f h}$.

Proof. Every sheaf $F$ satisfies the condition on the presheaf $P$ of the Lemma, since the induced map $L S p(k) \rightarrow L S p(L)$ is an isomorphism of sheaves if $L / k$ is any purely inseparable extension $[8,3.2 .5]$. This condition is closed under the formation of presheaf colimits. Every sheaf epimorphism is the coequalizer of its kernel pair, so it follows that the functor $\operatorname{hom}(L S p(k)$, ) takes colimits of sheaves to colimits in the set category.

Corollary 3. Suppose that $f: X \rightarrow Y$ is a local weak equivalence of simplicial sheaves on $\left(\left.S c h\right|_{k}\right)_{q f h}$, where $k$ is separably closed. Then the induced map $f$ : $X(k) \rightarrow Y(k)$ is a weak equivalence of simplicial sets.

Proof. The functor hom $\left(L S p(k)\right.$, ) preserves the $E x^{\infty}$ construction, and takes trivial local fibrations to trivial fibrations.

Corollary 4. Suppose that $f: X \rightarrow Y$ is a local weak equivalence of simplicial sheaves on $\left(\left.S c h\right|_{k}\right)_{q f h}$, where $k$ is separably closed. Suppose that $V$ is a finite $k$-scheme. Then the induced map $f: X(V) \rightarrow Y(V)$ is a weak equivalence of simplicial sets.

Proof. The objects $X$ and $Y$ are simplicial sheaves, so it suffices to assume that $V$ is connected. Then $V=S p(A)$ where $A$ is a finite local $k$-algebra with residue field $L$ which is purely inseparable over $k$ (recall that $k$ is separably closed). The maps $S p(L) \rightarrow S p(A)$ and $S p(L) \rightarrow S p(k)$ are universal homeomorphisms, so that the map $f: X(V) \rightarrow Y(V)$ is isomorphic to $f: X(k) \rightarrow Y(k)$. Now use Corollary 3.

Suppose that $T=S p(\mathcal{O})$, where $\mathcal{O}$ is a Noetherian strictly local Hensel ring. Let Fin $_{T}$ be the category of schemes which are finite over $T$, with finite surjective maps as coverings. Note that all finite morphisms $Z \rightarrow S p(\mathcal{O})$ are of the form $\sqcup_{i \in I} S p\left(\mathcal{O}_{i}\right) \rightarrow S p(\mathcal{O})$ where $I$ is a finite set, and each $\mathcal{O}_{i}$ is a strict local ring which is finite over $\mathcal{O}$. Let $\gamma:\left(\left.S c h\right|_{T}\right)_{q f h} \rightarrow$ Fin $_{T}$ be the corresponding site map. Suppose that $\tilde{F}$ denotes the sheaf associated to a presheaf $F$ on Fin $_{T}$.

Lemma 5. Suppose that $F$ is a presheaf on $\left(\left.S c h\right|_{T}\right)_{q f h}$ with associated sheaf map $\eta: F \rightarrow L F$, then the induced map $\left(\gamma_{*} F\right)^{\sim} \rightarrow \gamma_{*} L F$ is an isomorphism of sheaves on Fin .

Proof. Voevodsky tells us $[8,3.4 .2]$ that that every qfh cover $p: Y \rightarrow S p(R)$ of the spectrum of a strictly local ring $R$ can be refined by a finite cover (his proof 
requires refinement by separated covers), in the sense that there is a diagram

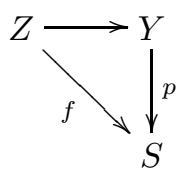

with $f$ finite and surjective. This means that the map of presheaves $\gamma_{*} F \rightarrow$ $\gamma_{*} L F$ is a local epi and a local monic of presheaves on Fin $_{T}$.

Corollary 6. Suppose that $f: X \rightarrow Y$ is a weak equivalence of simplicial sheaves on site $\left(\left.S c h\right|_{T}\right)_{q f h}$. Then the induced map $f_{*}: \gamma_{*} X \rightarrow \gamma_{*} Y$ of direct images is a local weak equivalence on Fin ${ }_{T}$.

Proof. The functor $\gamma_{*}$ is exact on sheaves on $\left(\left.S c h\right|_{T}\right)_{q f h}$ and in particular preserves sheaf epimorphisms. It follows that the restriction functor $\gamma_{*}$ commutes with the $E x^{\infty}$ construction up to isomorphism, and preserves trivial local fibrations. The desired result follows.

Corollary 7. Under the hypotheses of the previous lemma, the functor $\gamma_{*}$ preserves globally fibrant models of simplicial sheaves on $\left(\left.S c h\right|_{T}\right)_{q f h}$.

Proof. Suppose that $f: X \rightarrow Y$ is a local weak equivalence of simplicial sheaves on $\left(\left.S c h\right|_{T}\right)_{q f h}$, where $Y$ is globally fibrant. Then $\gamma_{*} Y$ is globally fibrant by formal nonsense, and the induced map $\gamma_{*} f: \gamma_{*} X \rightarrow \gamma_{*} Y$ is a local weak equivalence, by the previous corollary.

Proposition 8. Suppose that $A$ is a simplicial set consisting of finitely many simplices in each degree, and let $i: \Gamma^{*} A \rightarrow W$ be a globally fibrant model for the constant simplicial sheaf $\Gamma^{*} A$ on $\left(\left.S c h\right|_{T}\right)_{q f h}$. Then the induced map $A \rightarrow W(\mathcal{O})$ is a weak equivalence of simplicial sets.

Proof. First of all, there is an isomorphims

$$
\Gamma^{*} A(U) \cong \operatorname{hom}\left(\pi_{0} U, A\right) .
$$

This follows from $[8,3.2 .12]$, which implies that there is an isomorphism

$$
\operatorname{hom}_{T}\left(U, \sqcup_{A} T\right) \cong \operatorname{hom}\left(L(U), L\left(\sqcup_{A} T\right)\right)
$$

since $\sqcup_{A} T \rightarrow T$ is étale and of finite type over $T$, while it is not hard to show that $L\left(\sqcup_{A} T\right) \cong \Gamma^{*} A$ by comparing maps into arbitrary sheaves. It follows in particular that the canonical map $A \rightarrow \Gamma^{*} A(\mathcal{O})$ is an isomorphism of simplicial sets (since $\mathcal{O}$ is local, its assocated scheme is topologically connected).

Suppose that $x: S p(k) \rightarrow S p(\mathcal{O})$ denotes the residue map. Then it also follows that the canonical map

$$
\Gamma^{*} A \rightarrow x_{*} x^{*} \Gamma^{*} A
$$


restricts to an isomorphism on the finite site $\operatorname{Fin}_{T}$ for $T=S p(\mathcal{O})$. Note that $x^{*} \Gamma^{*} A$ is isomorphic to the constant sheaf $\Gamma^{*} A$ on $\left(\left.S c h\right|_{k}\right)_{q f h}$.

Suppose that $\alpha: \Gamma^{*} A \rightarrow W$ is a globally fibrant model for $\Gamma^{*} A$ on the site $\left(\left.S c h\right|_{T}\right)_{q f h}$. Then the pull back $x^{*} \Gamma^{*} A \rightarrow x^{*} W$ is a local weak equivalence. Choose a globally fibrant model $j: x^{*} W \rightarrow Z$ for $x^{*} W$ on $\left(\left.S c h\right|_{k}\right)_{q f h}$. It follows from Corollary 3 that the maps in sections

$$
x^{*} \Gamma^{*} A(V) \rightarrow x^{*} W(V) \rightarrow Z(V)
$$

are weak equivalences of simplicial sets for all finite $k$-schemes $V$, by Corollary 4.

The composite map

$$
\Gamma^{*} A \rightarrow x_{*} x^{*} \Gamma^{*} A \rightarrow x_{*} x^{*} W \rightarrow x_{*} Z
$$

therefore restricts to a pointwise weak equivalence on the site Fin $_{T}$, while at the same time $x_{*} Z$ is globally fibrant, so its restriction to the finite site is globally fibrant. Now use Corollary 7 to see that the map $\Gamma^{*} A \rightarrow W$ restricts to a globally fibrant model on Fin $_{T}$, so that the map $\gamma_{*} W \rightarrow \gamma_{*} x_{*} Z$ is a pointwise weak equivalence. The map $\gamma_{*} \Gamma^{*} A \rightarrow \gamma_{*} W$ is therefore a pointwise weak equivalence, so that the map $A \rightarrow W(\mathcal{O})$ in global sections is a weak equivalence of simplicial sets.

Corollary 9. Under the conditions of Proposition 8, there is a bijection

$$
H_{q f h}^{1}(\mathcal{O}, G)=*
$$

Suppose that $X$ is a Noetherian scheme and that $\mathcal{O}_{x}$ is the strict henselization of a geometric point $x: S p(K) \rightarrow X$ associated to some algebraically closed field $K$. Write $T_{x}=S p\left(\mathcal{O}_{x}\right)$. Then the site $\operatorname{Fin}_{T_{x}}$ is the filtered colimit of the sites Fin $_{U}$ for all étale neighbourhoods

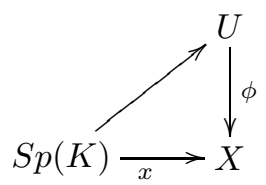

(where $\phi$ is étale) in the sense that the following conditions are satisfied:

1) for every morphism

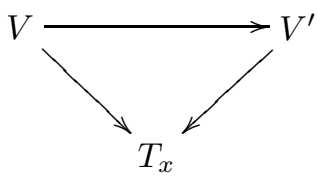

of schemes finite over $T_{x}$ there is a morphism

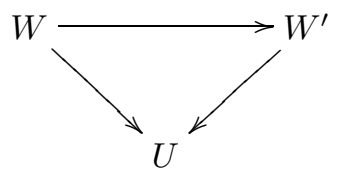


of finite schemes over $U$ which pulls back to the original diagram over $T_{x}$ up to isomorphism

2) any two maps

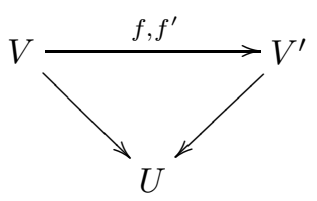

of $\left(\left.S c h\right|_{U}\right)_{\text {fin }}$ which pull back to the same morphism

$$
T_{x} \times_{U} V \rightarrow T_{x} \times_{U} V^{\prime}
$$

must pull back to the same morphism

$$
U^{\prime} \times_{U} V \rightarrow U^{\prime} \times_{U} V
$$

in $\left(\left.S c h\right|_{U^{\prime}}\right)_{f i n}$, for some refinement $\phi^{\prime}=\phi \psi$

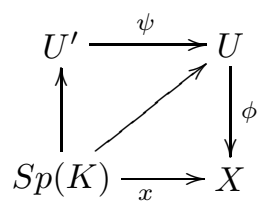

3) any finite surjective covering map $Y \rightarrow X$ of finite schemes over $T_{x}$ is isomorphic to the image under pullback of some covering $Y^{\prime} \rightarrow X^{\prime}$ of finite schemes over some $U$.

To verify the claim, it's harmless to suppose that the schemes $U$ appearing in the affine neighbourhoods $\phi$ are affine with $U=S p(R)$, by cofinality. Then all conditions follow from the fact that $\mathcal{O}_{x}$ is a filtered colimit of the rings $R$, together with the observation that integral extensions lift to the étale neighbourhood system.

The fibred finite site $\operatorname{Fin}_{D_{x}}$ for the diagram $D_{x}$ of étale neighbourhoods of $x: S p(K) \rightarrow X$ has for objects all diagrams

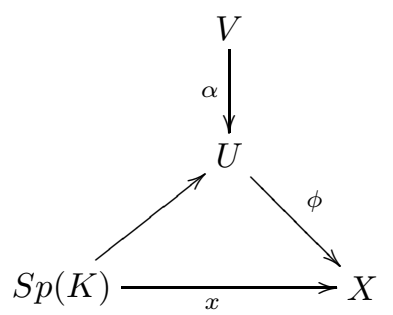


with $\alpha \in$ Fin $_{U}$. The morphisms of the category can be identified with all diagrams of the form

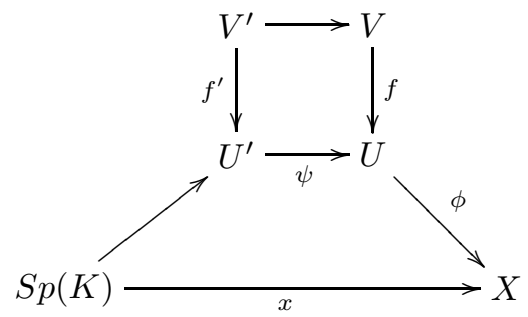

where $\psi$ is étale. Then pullback defines a functor

$$
\pi: \text { Fin }_{D_{x}} \rightarrow \text { Fin }_{T_{x}}
$$

in the obvious way, and this functor induces a restriction functor

$$
\pi_{*}: \operatorname{Pre}\left(\operatorname{Fin}_{T_{x}}\right) \rightarrow \operatorname{Pre}\left(\operatorname{Fin}_{D_{x}}\right)
$$

on the corresponding categories of presheaves.

Lemma 10. Suppose that $F$ is a sheaf on Fin $D_{D_{x}}$. Then the Kan extension $\pi^{p} F$ is a sheaf on Fin $_{T_{x}}$.

Proof. Suppose that $\gamma: W \rightarrow T_{x}$ is an object of Fin $_{T_{x}}$. Then, since $\operatorname{Fin}_{T_{x}}$ is a filtered colimit of the sites Fin $_{U}$ arising from étale neighbourhoods $U$, the set $\pi^{p} F(\gamma)$ is a filtered colimit of sets $F(\psi)$ arising from diagrams of the form

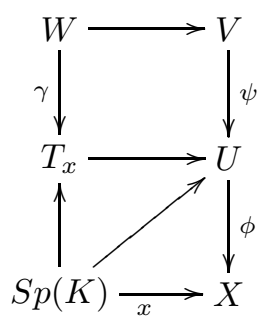

The collection of all $\psi$ which induce isomorphisms

$$
W \cong T_{x} \times_{U} V
$$

determines a cofinal full subcategory of this diagram category. If $\psi$ is a fixed object which induces such an isomorphism, then the collection of all morphisms

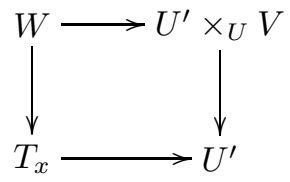


is again cofinal. For any finite surjective covering $Y \rightarrow W$ there is a finite surjective cover $Y^{\prime} \rightarrow V$ over some $U$ which pulls back to $Y \rightarrow W$ up to isomorphism. The sequences

$$
F\left(U^{\prime} \times_{U} V\right) \rightarrow F\left(U^{\prime} \times_{U} Y^{\prime}\right) \rightrightarrows F\left(U^{\prime} \times_{U} Y^{\prime} \times_{V} Y^{\prime}\right)
$$

are exact, and their filtered colimit is the sequence

$$
\pi^{p} F(W) \rightarrow \pi^{p} F(Y) \rightrightarrows \pi^{p} F\left(Y \times_{W} Y\right)
$$

which is therefore exact.

Note that one of the arguments in the previous proof specializes to the assertion that there is an isomorphism

$$
\pi^{p} F\left(T_{x}\right) \cong \underset{\phi}{\lim } F(U)
$$

where the (filtered) colimit is indexed over the étale neighbourhoods $\phi: U \rightarrow X$ of $x$.

The following diagram commutes up to isomorphism

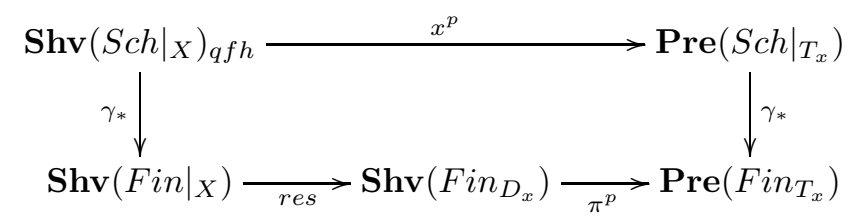

In effect, suppose given a diagram

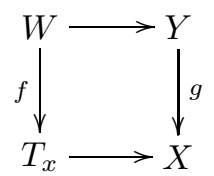

where $f$ is finite and $g$ is of finite type. Then there is an affine étale neighbourhood $U$ of $x$ and a finite map $U^{\prime} \rightarrow U$ such that $T_{x} \times_{U} U^{\prime} \cong W$ over $T_{x}$ such that the diagram factors as

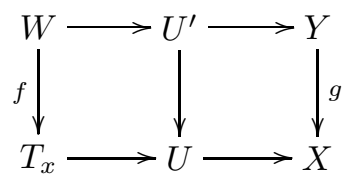

(see [5, p.71]). But then the colimiting diagram which defines $\left.\pi^{p} F\right|_{D_{x}}$ is cofinal in the diagram defining $x^{p} F$ at the finite morphism $f: W \rightarrow T_{x}$.

We have assembled what we need to prove the following: 
Lemma 11. Suppose that $F$ is a sheaf on $\left(\left.S c h\right|_{X}\right)_{q f h}$ where $X$ is a Noetherian scheme, suppose that $x: S p(K) \rightarrow X$ is a geometric point with corresponding strict Hensel ring $\mathcal{O}_{x}$, and let $T_{x}=S p\left(\mathcal{O}_{x}\right)$. Let $x$ also denote the induced map $x: T_{x} \rightarrow X$. Then there is a bijection

$$
x^{*}(F)\left(T_{x}\right)=\underline{\lim } F(U),
$$

where the filtered colimit is indexed over the étale neighbourhoods $U$ of $x$ in $X$.

Proof. The sheaf $x^{*} F$ is the sheaf associated to the presheaf $x^{p} F$, so that $\gamma_{*} x^{*} F$ is the sheaf associated to the presheaf $\gamma_{*} x^{p} F$, by Lemma 5. But then the diagram (1) commutes, and $\left.\pi^{p} F\right|_{D_{x}}$ is a sheaf by Lemma 10 .

Theorem 12. Suppose that $X$ is a Noetherian scheme and that $G$ is a sheaf of groups for the étale topology which is locally finite and constant. Then the associated sheaf map $\eta: G \rightarrow L G$ for the qfh topology is an isomorphism of étale sheaves, and the canonical function

$$
H_{e t}^{1}(X, G) \rightarrow H_{q f h}^{1}(X, G)
$$

is a bijection.

Proof. Supppose that $H$ is finite and constant for the étale topology. Then the associated sheaf map $\eta: H \rightarrow L H$ is an isomorphism, as in the proof of Proposition 8. The map $\eta: G \rightarrow L G$ is locally isomorphic to morphisms of the form $\eta: H \rightarrow L H$ for the étale topology, and is therefore an isomorphism of étale sheaves as well.

It suffices to show that every qfh $G$-torsor $P$ over a $X$-scheme $Y$ of finite type trivializes over an étale cover. If so, then the qfh stack completion map $B G \rightarrow B S t_{q f h} G[3]$ is a local weak equivalence for the étale topology. It follows that the map $B S t_{e t} G \rightarrow B S t_{q f h} G$ (of étale globally fibrant simplicial sheaves) is a pointwise equivalence. In particular, the map

$$
\pi_{0} B S t_{e t} G(X) \rightarrow \pi_{0} B S t_{q f h} G(X)
$$

is a bijection. This map coincides with the induced function in non-abelian $H^{1}$.

The problem is local for the étale topology, so we can assume that the sheaf of groups $G$ is constant and finite. Suppose that $x: S p(K) \rightarrow Y$ is a geometric point of $Y$. Then $x^{*} P$ is a qfh $G$-torsor on $T_{x}$, and so $x^{*} P$ has a global section by Corollary 9 . Then Lemma 11 implies that $P(U)$ has a section for some étale neighbourhood $U$ of $x$. The scheme $Y$ is Noetherian, so it follows that there is a covering of $Y$ consisting of finitely many étale maps $U_{i} \rightarrow Y$ such that $P$ is trivial over all members of the cover. 


\section{References}

[1] A. Grothendieck, Étude Locale des Schémas et des Morphisms de Schémas, Publ. Math. IHES 28 (1966). more to come.

[2] A. Grothendieck. Revetements étale et groupe fondamental (SGA1), Lecture Notes in Math. 224, Springer, Heidelberg (1971).

[3] J.F. Jardine, Stacks and the homotopy theory of simplicial sheaves, Preprint (2000).

[4] J.F. Jardine, The separable transfer, Preprint (2000).

[5] J.S. Milne, Etale Cohomology, Princeton University Press, Princeton (1980).

[6] A. Suslin and V. Voevodsky, Singular homology of abstract algebraic varieties, Invent. math. 123 (1996), 61-103.

[7] A. Suslin and V. Voevodsky, Relative cycles and Chow sheaves, Annals of Math. Studies 143 (2000), 10-86.

[8] V. Voevodsky, Homology of schemes, Selecta Mathematica, New Series bf 2(1) (1996), 111-153. 